International Journal of Biomedicine | June 2019 - Volume 9, Issue Suppl_1: Abstracts From the Second Russian International Conference "Cryo-electron microscopy 2019: achievements and prospects"

POSTER ABSTRACT PRESENTATIONS

SESSION TITLE: STRUCTURE AND FUNCTIONS OF THE TRANSCRIPTION AND TRANSLATION APPARATUS OF THE CELL

DOI: 10.21103/IJBM.9.Suppl_1.P20

\title{
Abstract P-20: Linker Histone H1: The Interplay between Chromatosome Stability, Oncomutations and Post-Translational Modifications
}

\author{
Mikhail V. Bass, Grigoriy A. Armeev, Alexey K. Shaytan \\ Department of Bioengineering, School of Biology, Moscow State University, Moscow, Russia
}

Background: Variations in the sequence of linker histone (LH) H1 play an important role in the modulation of chromatin functioning. Meanwhile, an effect of LH variations on the chromatin structure remains unclear. The most common mutations of the core histones are located in their globular domains (GD) and many of them coincide with post-translational modifications (PTM) sites. It is known that five mutations in GD of Drosophila H1 can change LH position in the chromatosome from "off-dyad" to "ondyad. Given polymorphism of chromatosome structure, an investigation of the effect of different LH variants on the chromatosome structure is extremely relevant.

Methods: Amino acid sequences of human LH H1.2, H1.4, and H1.5 were obtained from HistoneDB database. For homology modeling, the crystal structure of the chromatosome was used (PDB ID 4qlc) as a template. Post-translational modifications in the globular domain of linker histone H1, listed in the UniProt database, were considered for analysis. In order to analyze potential driver mutations, oncomutations of LH that were reported in the COSMIC database more than once were analyzed. Using FoldX software based on an empirical force field we measured the difference in binding free energy between the two states (1):

$$
\Delta \Delta G_{\text {bind }}=\Delta G_{2}-\Delta G_{1}
$$

where $\Delta G_{2}$ and $\Delta G_{1}$ are the binding free energies for a complex with or without a mutation (or PTM), respectively. For PTMs that could not be handled by FoldX, the screening of their positions for potential steric constraints was done using UCSF Chimera by changing the torsion angles of the modified side chains.

Results: Using FoldX we have estimated binding energy differences for 45 missense oncomutations and 27 PTM of human LH H1.2, H1.4 and H1.5. Oncomutations S58F and S104F of LH H1.2 manifested significant binding energy changes (2.33 and $4.10 \mathrm{kcal} / \mathrm{mol}$, respectively). These energies, however, are less than the experimentally measured free energy of dissociation of $\mathrm{H} 1$ in the 
presence of $0.2 \mathrm{M} \mathrm{NaCl}$, which is $8.5 \mathrm{kcal} / \mathrm{mol}$. Screening of PTM position allowed us to select variations of LH H1, which increase or decrease stability of the chromatosome.

Conclusion: We estimated the effect of PTM and oncomutations in GD of human LH on the chromatosome stability. PTM sites, in which additional positioning contacts between DNA and LH emerged, were determined. On the basis of the analysis, we can suggest that certain LH oncomutations may considerably change its binding affinity to nucleosome, potentially, affecting chromatin structure.

Key Words: nucleosome $\bullet$ chromatin $\bullet$ free energy calculations $\bullet$ histones

Sources of Funding: This work was supported by the Russian Science Foundation (project no. 19-7430003).

International Journal of Biomedicine. 2019;9 Suppl 1: S25. doi: 10.21103/IJBM.9.Suppl_1.P20

(C)2019 International Medical Research and Development Corporation 